\title{
Comprehensive psychological intervention to improve outcome in functional gastrointestinal disorder: a cohort study
}

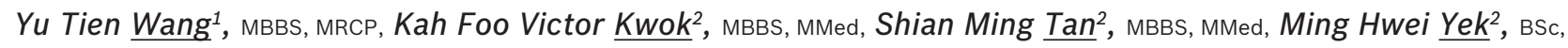
Wai Choung Ong $^{1}$, MBBS, MMed, Sylvaine Barbier ${ }^{3}$, MSc, Beng Yeong $\underline{\mathrm{Ng}}^{2}$, MBBS, MMed

INTRODUCTION Patients with functional gastrointestinal disorders (FGIDs) have a decreased quality of life (QoL). Psychological illnesses are strongly associated with FGIDs. This study examined the effect of a comprehensive psychological intervention programme designed for refractory FGID patients.

METHODS Refractory FGID patients at a tertiary gastroenterology unit were encouraged to participate in a psychological intervention programme, which included screening for anxiety and depression in patients, educating patients and physicians on FGIDs, and providing early access to psychiatric consultation for patients with significant psychological illnesses. The duration of follow-up was six months. Outcomes were measured using the Irritable Bowel Syndrome-QoL (IBS-QoL) instrument and the EuroQol five dimensions (EQ-5D) questionnaire.

RESULTS A total of 1,189 patients ( $68 \%$ female, $80 \%$ Chinese, mean age 48.6 years) participated in the programme. Among these participants, $51 \%$ had a significant psychological disorder (Hospital Anxiety and Depression Scale [HADS] anxiety or depression score > 7). These participants had a significantly poorer QoL (IBS-QoL and EQ-5D, both $p<$ 0.0001), and were more likely to be single or English-speaking, as compared to the participants without psychological disorders. Participants who completed $\geq 3$ months of follow-up $(n=906)$ showed significant and durable improvement. High baseline HADS anxiety score predicted improvement $(p<0.001)$, with participant IBS-QoL and EQ-5D SCores decreasing over time.

CONCLUSION The intervention programme was associated with a clinically meaningful improvement in the QoL of patients with refractory FGIDs. High baseline anxiety was predictive of improvement.

Keywords: anxiety, irritable bowel syndrome, psychological intervention

\section{INTRODUCTION}

Functional gastrointestinal disorders (FGIDs) are defined by the presence of chronic and bothersome gastrointestinal symptoms (e.g. abdominal pain and altered bowel movement) in patients with no structural abnormalities. These disorders, which include irritable bowel syndrome (IBS), functional dyspepsia, gastrooesophageal reflux disease and constipation, have a high disease burden. For example, IBS, which is a chronic condition that affects $10 \%-20 \%$ of the global population, ${ }^{(1)}$ has a significant impact on a patient's quality of life $(\mathrm{QoL})^{(2)}$ comparable to that of chronic renal failure $^{(3)}$ or ischaemic heart disease. ${ }^{(4)}$ The economic burden of IBS is also significant. In the United States alone, IBS care accounts for 3.6 million physician visits per year and over USD 20 billion in both direct and indirect expenditures. ${ }^{(5)}$

While the cause of FGIDs is multifactorial, psychological factors (manifesting as conditions like depression, and anxiety and somatisation disorders) are known to play an important role. ${ }^{(6,7)}$ Previous studies have shown that there is a strong association between FGIDs and psychiatric illnesses. ${ }^{\left({ }^{8-10)}\right.}$ However, it is challenging to address the psychological factors contributing to FGIDs in a busy gastroenterology unit. Psychological factors may be ignored or, even when they are identified, poorly managed due to time limitations or the gastroenterologist's lack of psychological training and/or awareness of the association between FGID and psychiatric conditions. As patients are often unaware of this association, they are unlikely to volunteer information regarding their psychological history. The negative stigma associated with psychiatric issues may also result in a patient's reluctance to discuss them, even when directly questioned. Furthermore, patients with a diagnosed psychiatric condition may face a long wait for a psychiatrist consultation. They may also be referred to a general psychiatrist who lacks FGID experience, resulting in the condition being managed in isolation from their FGIDs. These factors result in unsatisfactory FGID management, unnecessary investigations, frequent clinic consultations and in more severe cases, emergency department visits and hospitalisation. ${ }^{(5)}$

While the efficacy of individual psychological and psychopharmacological intervention in FGIDs is well described, ${ }^{(11)}$ the literature offers little on its application en masse in a clinical setting. Thus, the primary aim of the present study was to investigate the effect of the implementation of a comprehensive psychological intervention programme, which encompassed screening, education and psychiatrist intervention, in a highvolume tertiary gastroenterology unit. The secondary aims were

${ }^{1}$ Department of Gastroenterology and Hepatology, ${ }^{2}$ Department of Psychiatry, Singapore General Hospital, ${ }^{3}$ Centre for Quantitative Medicine, Duke-NUS Graduate Medical School, Singapore

Correspondence: Dr Yu Tien Wang, Consultant, Department of Gastroenterology and Hepatology, Singapore General Hospital, 20 College Road, Singapore 169856. wang.yu.tien@sgh.com.sg 
to: (a) identify baseline characteristics that predict improvement through the programme; (b) evaluate patient acceptance of the programme; and (c) evaluate the effect of psychiatrist intervention in patients with significant psychological disorders.

\section{METHODS}

The present study was conducted in the gastroenterology unit with the highest clinical volume in Singapore $(48,000$ patients per year). The study conforms to the provisions of the Declaration of Helsinki (as revised in Tokyo 2004) and was approved by the SingHealth Centralised Institutional Review Board, Singapore.

At the introduction to the programme, all unit gastroenterologists were educated on the role of psychological disorders in FGIDs and the importance of their management. Educational posters describing the importance of psychological disorders in FGIDs and availability of the intervention programme were also displayed in patient waiting areas. All FGID patients who were dissatisfied with their first-line treatment, as determined by their attending gastroenterologist, were considered refractory for the purposes of the programme and the study. These refractory patients were invited to participate in the programme. Upon referral, trained coordinators interviewed the participants, explained the rationale for the screening (i.e. to provide early psychological intervention for FGID participants) and obtained written informed consent.

Psychological screening was performed using the Hospital Anxiety and Depression Scale (HADS), ${ }^{(12)}$ a validated instrument for detecting anxiety and depression in patients with medical illness. A score of $>7$ on either the HADS anxiety or depression measure was considered significant; participants with these scores were offered early referral (within two weeks) to a psychiatrist who had specific knowledge on the association between psychiatric conditions and FGIDs to examine whether the patient had an FGIDassociated psychiatric disorder. The attending gastroenterologists were informed of the HADS results and any planned referrals, and continued standard-of-care management during the study period.

Disease-specific QoL was measured using the IBS-QoL instrument, ${ }^{(13)}$ an extensively validated QoL measure for FGID. The IBS-QoL instrument contains 34 items, each graded on a five-point scale. The total score ranges from 0-100, with higher scores indicating improved QoL. Generic QoL was measured using the EuroQol five dimensions (EQ-5D) questionnaire. ${ }^{(14)}$ Its use in patients with FGIDs has previously been described. ${ }^{(15)}$ It consists of five items and each item is graded on three levels of response: (a) no problems, which is assigned a rating of 1 ; (b) some problems, which is assigned a rating of 2; and (c) severe problems, which is assigned a rating of 3 . These ratings are converted into a single QoL score using a calculator that is available from the EuroQol Group. In the present study, the United Kingdom value set ${ }^{(16)}$ was applied due to robust supporting evidence and the lack of an available local value set. EQ-5D scores for health states range from $-0.6-1$, with 1 representing full health, 0 representing death and a negative score representing a health state worse than death. Changes in the IBS-QoL and EQ-5D scores of the participants were assessed by comparing their baseline scores to their scores at three and six months post-intervention.
Receiver operating characteristic (ROC) curve analyses were conducted to identify the baseline factors that could predict an improvement in the IBS-QoL and EQ-5D scores. Clinically significant improvement was defined as a $20 \%$ increase in the patient's QoL scores from the baseline at three months post-intervention; this approximates to an absolute increase of 14 points in the IBS-QoL score, which has been established as indicating a clinically meaningful improvement. ${ }^{(17)}$ The effect of attending an early psychiatrist appointment was assessed by comparing the changes (from baseline to three and six months post-intervention) in the IBS-QoL and EQ-5D scores of eligible participants (i.e. with HADS anxiety or depression score $>7$ ) who attended the psychiatric consultation with the changes in the scores of eligible patients who chose not to attend the psychiatric consultation.

Patients who were referred to the intervention programme were interviewed by a trained coordinator at the earliest opportunity; $90 \%$ of the participants were screened within 24 hours of referral. Telephone screening was conducted for those who were not screened on the day of referral. Verbal translations of the questionnaires were provided when necessary. The trained coordinator provided the participants with information on FGID before conducting the psychological screening. Participants were educated on FGID symptoms, triggers and management, and the link between the manifestation of FGID symptoms and stress (one of the triggers) was highlighted. HADS, EQ-5D and IBS-QoL measures were administered to the participants who agreed to be screened. HADS was scored immediately after administration and participants were informed of their scores. Participants with a score of $>7$ for anxiety and/or depression were offered an appointment to consult with a psychiatrist, while those who declined early psychiatric intervention were provided with further psychoeducation on stress management.

Participants received an information handout (Appendix) and an explanation by the coordinator. They were informed of the manifestations of stress, including anticipatory worries, behaviours such as avoidance and bodily reactions such as gastrointestinal symptoms. Methods for managing stress were also described, including distraction, exercising, talking and deep breathing. Distraction entailed participants thinking of a word such as 'relax' and repeating it for ten minutes with their eyes closed. Exercising and talking to someone were encouraged to improve mood and for stress release. The coordinator also demonstrated a deep breathing method, whereby the abdomen would expand during inhalation and contract during exhalation. Finally, participants were taught to evaluate the validity of their stressful thoughts by gathering evidence for and against them. This was carried out during the initial referral and took an average of 30-60 minutes.

For the participants who accepted early psychiatric intervention, a psychiatrist would perform a detailed patient history-taking and mental state examination during the consultation. During historytaking, the patient's stressors, symptoms and the resulting sociooccupational impairment would be assessed. If the participant was diagnosed with a psychiatric condition, the psychiatrist 
Table I. Baseline demographics of the participants $(n=1,189)$.

\begin{tabular}{|c|c|c|c|c|}
\hline \multirow[t]{2}{*}{ Variable } & \multicolumn{3}{|c|}{$\%$} & \multirow[t]{2}{*}{ p-value } \\
\hline & All $(n=1,189)$ & $\begin{array}{l}\text { HADS score }>7 \\
(n=603)\end{array}$ & $\begin{array}{c}\text { HADS score } \leq 7 \\
(n=586)\end{array}$ & \\
\hline Age $(y r)^{*}$ & $48.60 \pm 15.10$ & $47.90 \pm 15.10$ & $49.40 \pm 15.00$ & $<0.0001$ \\
\hline HADS depression score at baseline ${ }^{*}$ & $4.21 \pm 3.91$ & $4.55 \pm 4.06$ & $3.87 \pm 3.72$ & $<0.0001$ \\
\hline HADS anxiety score at baseline* & $7.29 \pm 4.58$ & $8.02 \pm 4.67$ & $6.54 \pm 4.35$ & $<0.0001$ \\
\hline IBS-QoL score* & $80.60 \pm 19.00$ & $74.80 \pm 21.00$ & $86.50 \pm 14.70$ & $<0.0001$ \\
\hline EQ-5D score* & $0.69 \pm 0.23$ & $0.63 \pm 0.25$ & $0.76 \pm 0.20$ & $<0.0001$ \\
\hline HADS depression score $>7$ & 19 & - & - & - \\
\hline HADS anxiety score $>7$ & 47 & - & - & - \\
\hline HADS depression score $>7$ or anxiety score $>7$ & 51 & - & - & - \\
\hline HADS depression score $>7$ and anxiety score $>7$ & 16 & - & - & - \\
\hline Gender & & & & 0.9935 \\
\hline Male & 32 & 32 & 32 & \\
\hline Female & 68 & 68 & 68 & \\
\hline Ethnicity & & & & 0.3764 \\
\hline Chinese & 80 & 78 & 81 & \\
\hline Malay & 6 & 7 & 6 & \\
\hline Indian & 10 & 10 & 9 & \\
\hline Others & 4 & 5 & 3 & \\
\hline Education level & & & & 0.0862 \\
\hline No formal education & 5 & 5 & 5 & \\
\hline Primary $(1-6$ yr) & 16 & 14 & 17 & \\
\hline Secondary (10-12 yr) & 32 & 32 & 35 & \\
\hline Tertiary ( $\geq 12 \mathrm{yr}$ ) & 46 & 50 & 43 & \\
\hline Literate & & & & 0.2304 \\
\hline Yes & 93 & 92 & 93 & \\
\hline No & 7 & 9 & 7 & \\
\hline Language of interview & & & & 0.0368 \\
\hline English & 66 & 71 & 64 & \\
\hline Mandarin & 31 & 25 & 33 & \\
\hline Malay & 1 & 1 & 1 & \\
\hline Tamil & 0 & 0 & 1 & \\
\hline Others & 2 & 2 & 2 & \\
\hline Marital status & & & & 0.0070 \\
\hline Single & 29 & 32 & 25 & \\
\hline Married/cohabiting & 60 & 58 & 64 & \\
\hline Divorced/separated & 6 & 7 & 5 & \\
\hline Widowed & 5 & 3 & 6 & \\
\hline Housing type & & & & 0.5020 \\
\hline Small HDB apartment & 6 & 7 & 5 & \\
\hline Medium HDB apartment & 49 & 48 & 49 & \\
\hline Large HDB apartment & 28 & 29 & 27 & \\
\hline Private apartment/condominium & 10 & 10 & 11 & \\
\hline Landed house & 8 & 7 & 8 & \\
\hline Employment & & & & 0.2408 \\
\hline Retired & 13 & 10 & 14 & \\
\hline Self-employed & 3 & 4 & 3 & \\
\hline Part-time/temporary & 8 & 7 & 8 & \\
\hline Full-time & 52 & 54 & 52 & \\
\hline Unemployed/housewife & 21 & 21 & 21 & \\
\hline Student & 3 & 4 & 2 & \\
\hline Religion & & & & 0.3110 \\
\hline Taoism/Buddhism & 42 & 43 & 40 & \\
\hline
\end{tabular}




\begin{tabular}{|c|c|c|c|c|}
\hline \multirow[t]{2}{*}{ Variable } & \multicolumn{3}{|c|}{$\%$} & \multirow[t]{2}{*}{ p-value } \\
\hline & All $(n=1,189)$ & $\begin{array}{c}\text { HADS score }>7 \\
(n=603)\end{array}$ & $\begin{array}{c}\text { HADS score } \leq 7 \\
(n=586)\end{array}$ & \\
\hline Christianity & 26 & 24 & 26 & \\
\hline Hinduism & 8 & 8 & 7 & \\
\hline Islam & 8 & 10 & 8 & \\
\hline Others & 16 & 16 & 19 & \\
\hline Monthly income (SGD) & & & & 0.0667 \\
\hline$<1,000$ & 43 & 42 & 41 & \\
\hline $1,000-2,999$ & 30 & 29 & 35 & \\
\hline $3,000-5,999$ & 20 & 22 & 17 & \\
\hline $6,000-9,999$ & 6 & 6 & 5 & \\
\hline$\geq 10,000$ & 2 & 2 & 2 & \\
\hline
\end{tabular}

*Data presented as mean \pm standard deviation. EQ-5D: EuroQol five dimensions questionnaire; HADS: Hospital Anxiety and Depression Scale; HDB: Housing and Development Board; IBS-QoL: Irritable Bowel Syndrome-Quality of Life instrument

Table II. Comparison of IBS-QoL and EQ-5D scores over time.

\begin{tabular}{|c|c|c|c|c|c|c|c|}
\hline \multirow[t]{2}{*}{ Measure } & \multicolumn{3}{|c|}{ Mean \pm standard deviation } & \multirow[t]{2}{*}{ p-value* } & \multicolumn{2}{|c|}{ Mean \pm standard deviation } & \multirow[t]{2}{*}{ p-value } \\
\hline & $\begin{array}{l}\text { Baseline } \\
(\mathrm{n}=906)\end{array}$ & $\begin{array}{l}\text { At 3-mth follow-up } \\
\qquad(\mathrm{n}=906)\end{array}$ & Difference & & $\begin{array}{l}\text { At 6-mth follow-up } \\
\qquad(\mathrm{n}=550)\end{array}$ & Difference & \\
\hline EQ-5D & $0.70 \pm 0.23$ & $0.81 \pm 0.20$ & $0.11 \pm 0.25$ & $<0.001$ & $0.82 \pm 0.21$ & $0.14 \pm 0.26$ & $<0.001$ \\
\hline
\end{tabular}

Data presented as mean \pm standard deviation. *Paired $t$-test (three months vs. baseline). ${ }^{\dagger}$ Paired $t$-test (six months vs. baseline). EQ-5D: EuroQol five dimensions questionnaire; IBS-QoL: Irritable Bowel Syndrome-Quality of Life instrument

Table III. Comparison of IBS-QOL and EQ-5D scores in patients with and without a significant psychological disorder.

\begin{tabular}{|c|c|c|c|c|c|c|c|c|c|}
\hline \multirow[t]{2}{*}{ Measure } & \multirow[t]{2}{*}{ HADS } & \multicolumn{3}{|c|}{ Mean \pm standard deviation } & \multirow[t]{2}{*}{ p-value* } & \multirow[t]{2}{*}{ HADS } & \multicolumn{2}{|c|}{ Mean \pm standard deviation } & \multirow[t]{2}{*}{ p-value* } \\
\hline & & $\begin{array}{l}\text { Baseline } \\
(n=906)\end{array}$ & $\begin{array}{l}\text { At 3-mth } \\
\text { follow-up } \\
(n=906)\end{array}$ & Difference & & & $\begin{array}{l}\text { At 6-mth } \\
\text { follow-up } \\
(n=550)\end{array}$ & Difference & \\
\hline \multirow[t]{2}{*}{ IBS-QoL } & $\leq 7(n=454)$ & $87.30 \pm 14.20$ & $91.40 \pm 12.60$ & $4.10 \pm 11.60$ & $<0.001$ & $\leq 7(n=265)$ & $92.50 \pm 12.20$ & $4.30 \pm 13.20$ & $<0.001$ \\
\hline & $>7(n=452)$ & $74.70 \pm 21.70$ & $83.30 \pm 20.20$ & $8.60 \pm 16.90$ & & $>7(n=285)$ & $84.00 \pm 20.30$ & $9.60 \pm 18.50$ & \\
\hline \multirow[t]{2}{*}{$E Q-5 D$} & $\leq 7(n=454)$ & $0.76 \pm 0.19$ & $0.85 \pm 0.17$ & $0.09 \pm 0.21$ & 0.016 & $\leq 7(n=265)$ & $0.87 \pm 0.15$ & $0.12 \pm 0.23$ & 0.060 \\
\hline & $>7(n=452)$ & $0.64 \pm 0.24$ & $0.77 \pm 0.22$ & $0.13 \pm 0.27$ & & $>7(n=285)$ & $0.78 \pm 0.24$ & $0.16 \pm 0.28$ & \\
\hline
\end{tabular}

*Paired $t$-test (HADS score > 7 vs. HADS score $\leq 7$ ). EQ-5D: EuroQol five dimensions questionnaire; HADS: Hospital Anxiety and Depression Scale; IBS-QoL: Irritable Bowel Syndrome-Quality of Life instrument

would explore the relationship between the patient's FGIDs and the psychiatric condition, and how it affected help-seeking behaviour. Treatment was biological and psychosocial. The most commonly prescribed medications were tricyclic antidepressants and selective serotonin reuptake inhibitors. The participants could also be treated with cognitive behavioural therapy. All participants who were referred to the programme were also encouraged to join a monthly FGID support group. Each session consisted of a 45-minute talk by an FGID specialist (gastroenterologist, psychiatrist, psychologist or dietician), followed by a 45-minute sharing session during which participants were encouraged to share their own experiences and offer support to one another. The support group sessions were facilitated by a psychologist and a trained coordinator. Paired $t$-tests were used to compare participants' IBS-QoL, EQ-5D and HADS anxiety baseline scores with their scores at three months and six months post-intervention. ROC curves were used to identify the cut-off point for each selected variable that provided the largest number of correct classifications (i.e. had the maximum sensitivity and specificity) to identify which participants had a better likelihood of improvement. SAS PROC LOGISTIC (SAS Institute's macro \%ROC version 1.7 and macro \%CALCSN) (Beckman Coulter Inc, San Diego, CA, USA) was used for ROC analyses. ${ }^{(18)}$ Longitudinal analysis of the association between the IBS-QoL score and the HADS anxiety score was performed using the generalised estimating equation (GEE) with the GENMOD procedure. ${ }^{(19)}$ This method ensures that the observations noted for a single participant (at baseline, and three and six months post-intervention) are correlated. The most accurate working correlation structure for the model was selected with the aid of the quasi-likelihood ratio.

If the participant underwent at least six months of follow-up, we also ran a subgroup analysis to determine the effect of early psychiatric consultation on the eligible participants (i.e. participants with a HADS anxiety or depression score $>7$ ). The demographic variables of the eligible participants who attended the early appointment with a psychiatrist were compared with those of the eligible participants who did not, using $t$-tests or chi-square tests, as appropriate. We then analysed the evolution of the IBS-QoL 
and EQ-5D scores. Paired $t$-tests were used to assess the difference within each group, while a $t$-test was used to assess the difference between the baseline score and the score at six months follow-up between the two groups. Tests for the appropriate assumptions were carried out and models were checked. All data was analysed using the Statistical Analysis System version 9.2 (SAS Institute, Cary, NC, USA) and the level of significance was set at $5 \%$.

\section{RESULTS}

A total of 1,219 patients were referred to the programme. However, ten patients declined to participate and 20 were excluded from analysis because they did not consent to the use of their data for research despite agreeing to participate in the programme. Thus, a total of 1,189 patients participated in the present study and had their data included in the analysis. Their mean age was 48.60 years; $68 \%$ were female and $80 \%$ were Chinese. The mean baseline HADS anxiety and depression scores were 7.29 and 4.21, respectively. 603 participants (51\%) had a HADS anxiety or depression score of $>7$. Participants with a significant psychological disorder had a significantly poorer QoL than those without a significant psychological disorder (IBS-QoL 74.80 vs. 86.50 and EQ-5D 0.63 vs. 0.76 , both $p<0.0001$ ). The participants with a significant psychological disorder were also more likely to be single or English-speaking compared to the participants without a psychological disorder (Table I).

Participants who completed at least three months of follow-up $(n=906)$ were included in our primary outcome analysis. Highly significant improvements were seen in both the IBS-QoL score (from a mean score of 80.98 to 87.36 at three months, and to 88.13 at six months, both $\mathrm{p}<0.001$ ) and EQ-5D score (from a mean score of 0.70 to 0.81 at three months, and to 0.82 at six months, both $p$ $<0.001$ ) (Table II). The improvement shown by participants with a significant psychological disorder was significantly greater than in those without a significant psychological disorder (Table III). A high baseline HADS anxiety score predicted improvement $(\mathrm{p}<$ 0.001), with programme participant IBS-QoL and EQ-5D scores decreasing over time (from 7.4 to 4.9 at three months and to 4.4 at six months, both $\mathrm{p}<0.001$ ).

In the ROC curve analysis to identify baseline factors that could be predictive of an improvement in participants' IBS-QoL and EQ5D scores, we found that the available variables at baseline had poor predictive value except for the HADS anxiety score, which yielded an area under the curve of $0.70(p<0.001)$ for improvement in the IBS-QoL. The cut-off point that had the maximum sensitivity (0.67) and specificity (0.64) was a HADS anxiety score of 9; that is, a participant with a baseline HADS anxiety score of $>9$ was more likely to have an improvement in IBS-QoL score after three months. The GEE indicated that the IBS-QoL score deteriorated with an increase in the HADS anxiety score: each one-unit increase in the HADS anxiety score led to a 1.63 reduction in the IBS-QoL score $(p<0.001)$. The IBS-QoL score improved with time spent in the intervention programme, increasing by an average of 0.44 each month $(p<0.001)$ (Table IV \& Fig. 1).

In the subgroup analysis of participants with a significant psychiatric disorder, there was no significant difference between
Table IV. Association between the study population's HADS anxiety score and IBS-QoL score over time $(n=906)$.

\begin{tabular}{|c|c|c|c|c|}
\hline Parameter & Estimate & $\begin{array}{l}\text { Standard } \\
\text { error }\end{array}$ & $\begin{array}{l}95 \% \text { confidence } \\
\text { limits }\end{array}$ & p-value \\
\hline Intercept & 93.38 & 0.73 & $(91.94 ; 94.81)$ & $<0.0001$ \\
\hline $\begin{array}{l}\text { HADS anxiety } \\
\text { score }\end{array}$ & -1.63 & 0.10 & $(-1.83 ;-1.44)$ & $<0.0001$ \\
\hline Month & 0.44 & 0.11 & $(0.22 ; 0.65)$ & $<0.0001$ \\
\hline
\end{tabular}

Analysis was performed using the Generalized Estimating Equation. Quasi-likelihood under the independence model criterion (QIC) = 2367.4; simplified QIC $=2365.0$. HADS: Hospital Anxiety and Depression Scale; IBS-QoL: Irritable Bowel Syndrome-Quality of Life instrument.

the IBS-QoL and EQ-5D scores of the group that received early psychiatric consultation and the group that did not. Both showed significant improvements that were of similar amplitude (Table V).

\section{DISCUSSION}

The comprehensive psychological intervention programme resulted in a significant improvement in the IBS-QoL and EQ-5D scores of refractory FGID patients in a high-volume gastroenterology unit; this improvement was durable over six months. Participants with high baseline HADS anxiety scores were found to be more likely to improve in the programme. The optimal cut-off was a baseline HADS anxiety score of $>9$. The programme was well accepted by the patients, with nearly all referred patients agreeing to undergo evaluation and education. However, only about half of the participants eligible for psychiatric intervention accepted the invitation. When the participants with significant psychological disorders were evaluated, we found that outcomes did not differ significantly between those who received psychiatric intervention and those who did not.

The improvement in the QoL of the patients with refractory FGIDs increased with time and corresponded to a decrease in anxiety levels. Although we did not have a control group, previous studies on the natural history of hospital-based FGID patients have shown that patient QoL does not improve significantly over time with standard-of-care management. ${ }^{(20)}$ Therefore, it is likely that the psychological intervention programme had a positive effect.

Psychological abnormality, particularly anxiety, is strongly associated with FGIDs. ${ }^{(6)}$ In fact, anxiety may be the primary cause of FGID symptoms in some patients. ${ }^{(8-10)}$ Anxiety has also been shown to be a strong predictor of healthcare-seeking behaviour among FGID patients. ${ }^{(21)}$ These findings are parallel to the findings of the present study, in which there was a high prevalence of psychological burden among the participants. We found that participants with psychological disorders had poorer QoL baselines. We also found that high anxiety levels were predictive of clinically significant improvement in the programme. In addition, the severity of participants' clinical condition, as reflected by IBS-QoL scores, showed reciprocal improvement as anxiety level improved; the GEE showed that a reduction in anxiety score is strongly associated with an improvement in QoL. These findings, together with those of the aforementioned studies, highlight the importance of psychological disorder treatment in FGID management. ${ }^{(22)}$ 


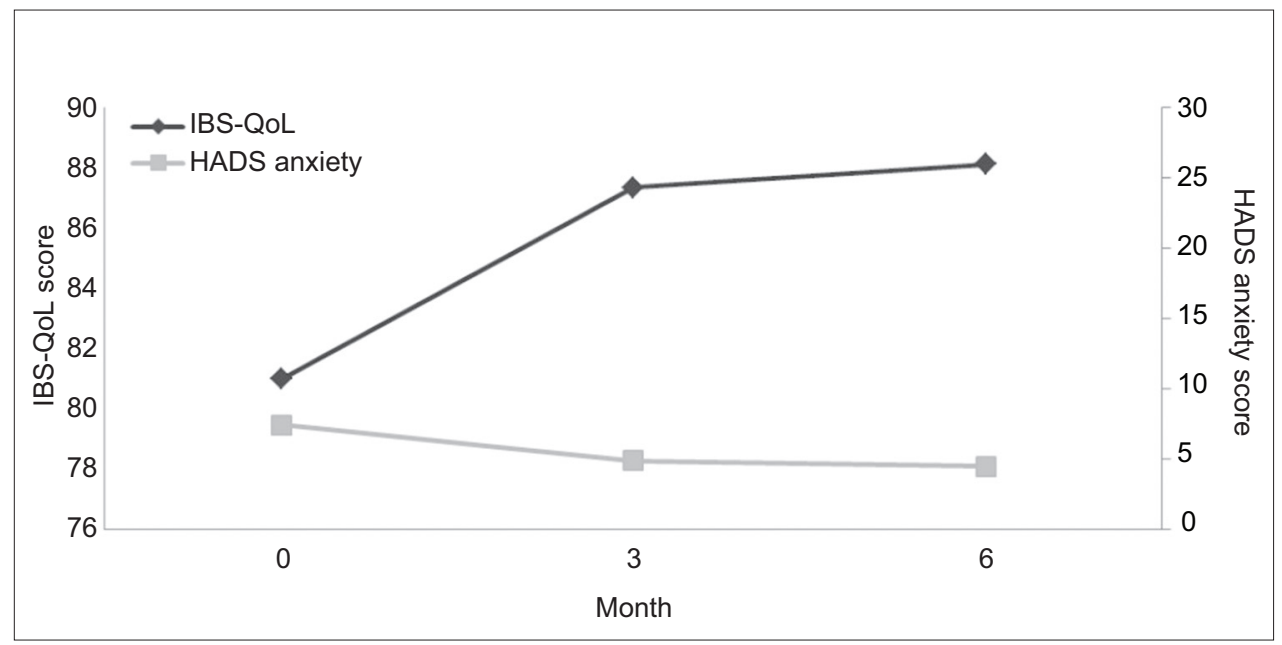

Fig. 1 Chart shows average change in Irritable Bowel Syndrome-Quality of Life (IBS-QoL) scores and Hospital Anxiety and Depression Scale (HADS) anxiety scores over time.

Table V. Comparison of the IBS-QoL scores of participants who received early psychiatric intervention $(n=216)$ and those who did not (i.e. education only) $(n=212)$.

\begin{tabular}{|c|c|c|c|c|c|}
\hline \multirow[t]{2}{*}{ Treatment received } & \multicolumn{3}{|c|}{ Mean \pm standard deviation } & \multirow{2}{*}{$\begin{array}{l}\text { p-value* } \\
\text { (within } \\
\text { group) }\end{array}$} & \multirow{2}{*}{$\begin{array}{c}\text { p-value } \\
\text { (between } \\
\text { groups) }\end{array}$} \\
\hline & $\begin{array}{c}\text { Baseline } \\
\text { IBS-QoL score }\end{array}$ & $\begin{array}{l}\text { IBS-QoL score at } \\
\text { 6-mth follow-up }\end{array}$ & Difference & & \\
\hline Psychiatric intervention & $78.26 \pm 20.67$ & $87.42 \pm 18.07$ & $9.19 \pm 17.77$ & $<0.001$ & 0.8152 \\
\hline Education only & $75.34 \pm 21.38$ & $83.91 \pm 21.25$ & $8.57 \pm 20.24$ & 0.001 & \\
\hline
\end{tabular}

Both groups of patients had a significant psychiatric disorder. *Paired $t$-test (baseline vs. six months). $t$-test of the difference in the differences (psychiatrist intervention vs. education only). IBS-QoL: Irritable Bowel Syndrome-Quality of Life instrument.

In the present study, we also attempted to further qualify whether early psychiatric consultations, which enable psychological or psychopharmacological treatment, were effective in improving outcome. We found no significant difference in the outcomes of the eligible participants who attended psychiatric consultation and those of the eligible participants who did not. Both groups showed marked improvement after the programme. Although this finding seems to suggest that the FGID patients with psychiatric disorders improved on conventional treatment alone (i.e. without psychological intervention), it is unlikely given that previous studies have shown that FGID patients on conventional treatment tend to deteriorate with time. ${ }^{(22)}$ Therefore, it is more likely that the other interventions in the present study's psychological intervention programme provided significant benefits to the participants who did not attend the psychiatric consultation, thus diluting the difference in outcome between them and participants who did attend the consultation. One such intervention was the psychoeducation that the coordinator administered to the participants who declined formal psychiatric consultation. Indeed, education and brief psychological therapy have been shown to benefit FGID patients. ${ }^{(23,24)}$ In addition, the programme was designed such that the primary gastroenterologist would be informed of any abnormal psychological screening results. This may have prompted the gastroenterologist to initiate psychopharmacological treatment, particularly for participants who did not attend psychiatric consultation.

In Singapore, previous community IBS data has shown nearequal gender distribution. ${ }^{(4,25)}$ However, $68 \%$ of the present study's cohort of hospital-based patients with refractory FGIDs (the majority had a diagnosis of IBS) were female. This suggests that women are more likely to have refractory FGID symptoms that require tertiary care consultation. This association may be explained by a concept known as gender role socialisation. ${ }^{(26)}$ For example, social norms dictate that women keep their bodily functions private and orderly. Therefore, women may experience more distress than men in response to bodily function disruptions (e.g. altered bowel habits) that commonly occur with FGIDs, prompting them to seek help from physicians.

In the present study, the income of the participants was significantly below the national average. More than $43 \%$ of our participants had a monthly income < SGD 1,000, compared to $25 \%$ in the general population, according to a national census. ${ }^{(27)}$ While this may be a reflection of the demographics of the patients who attend public health institutions, it may also indicate that low income is associated with refractory FGIDs. The association of FGIDs with lower household income has also been demonstrated in a Western study. ${ }^{(28)}$

Among the strengths of the present study were its large sample size and the clinical implementation of the programme in a busy gastroenterology unit. These qualities of the study mean that the results obtained are likely to be reproducible when the resources described are used. The patient interview, FGID education and psychoeducation that were conducted during the initial referral took 30-60 minutes on average. They were managed by one trained coordinator, who also managed the patient support group. 
One major weakness of the study was the lack of a control group. Comparisons could only be made with historical data from other hospital-based populations. In addition, the study used the referring physician's diagnosis of refractory FGIDs rather than established diagnostic criteria. This was due to the fact that the programme was introduced in the clinical setting to assist busy gastroenterologists and it was not feasible for the referring physicians to ensure that the patients fulfilled research diagnostic criteria prior to referral to the programme. Nonetheless, as the department advocates the use of regional guidelines ${ }^{(1,29)}$ in the management of FGIDs, participants in the study were unlikely to have organic diseases. Finally, as the participants were from a tertiary centre, the findings of the present study should not be generalised to primary or secondary care settings.

To conclude, although psychological illnesses are strongly associated with FGIDs, patients are seldom aware of this association and gastroenterologists often have no time to manage these psychological issues. The comprehensive psychological intervention programme implemented in our busy gastroenterology unit was effective in improving the outcomes of patients with refractory FGIDs. We found the programme to be particularly effective in FGID patients with high baseline anxiety levels. The effect was durable over time and the programme was well accepted by patients. The positive outcome may be related to an overall improvement in awareness of participants and physicians about the association between FGIDs and psychological factors, as well as the importance of managing the psychological issues. The use of a comprehensive psychological intervention programme that encompasses screening, education and psychiatric intervention should be considered for implementation in busy tertiary gastroenterology units that have a large burden of refractory FGIDs.

\section{ACKNOWLEDGEMENT}

The authors appreciate the medical editing assistance of Jon Kilner, MS, MA (Pittsburgh, Pennsylvania, USA).

\section{REFERENCES}

1. Gwee KA, Bak YT, Ghoshal UC, et al; Asian Neurogastroenterology and Motility Association. Asian consensus on irritable bowel syndrome. J Gastroenterol Hepatol 2010; 25:1189-205.

2. El-Serag HB, Olden K, Bjorkman D. Health-related quality of life among persons with irritable bowel syndrome: a systematic review. Aliment Pharmacol Ther 2002; 16:1171-85.

3. Gralnek IM, Hays RD, Kilbourne A, Naliboff B, Mayer EA. The impact of irritable bowel syndrome on health-related quality of life. Gastroenterology 2000; 119:654-60.

4. Wang YT, Lim HY, Tai D, et al. The impact of irritable bowel syndrome on health-related quality of life: a Singapore perspective. BMC Gastroenterol 2012; $12: 104$.

5. Everhart JE, Ruhl CE. Burden of digestive diseases in the United States part II: lower gastrointestinal diseases. Gastroenterology 2009; 136:741-54.

6. Sykes MA, Blanchard EB, Lackner J, Keefer L, Krasner S. Psychopathology in irritable bowel syndrome: support for a psychophysiological model.
J Behav Med 2003; 26:361-72.

7. Bennett EJ, Piesse C, Palmer K, et al. Functional gastrointestinal disorders: psychological, social, and somatic features. Gut 1998; 42:414-20.

8. Whitehead WE, Crowell MD, Robinson JC, Heller BR, Schuster MM. Effects of stressful life events on bowel symptoms: subjects with irritable bowel syndrome compared with subjects without bowel dysfunction. Gut 1992; 33:825-30.

9. Walker LS, Garber J, Smith CA, Van Slyke DA, Claar RL. The relation of daily stressors to somatic and emotional symptoms in children with and without recurrent abdominal pain. J Consult Clin Psychol 2001; 69:85-91.

10. Levy RL, Cain KC, Jarrett M, Heitkemper MM. The relationship between daily life stress and gastrointestinal symptoms in women with irritable bowel syndrome. J Behav Med 1997; 20:177-93.

11. Ford AC, Talley NJ, Schoenfeld PS, Quigley EM, Moayyedi P. Efficacy of antidepressants and psychological therapies in irritable bowel syndrome: systematic review and meta-analysis. Gut 2009; 58:367-78.

12. Zigmond AS, Snaith RP. The hospital anxiety and depression scale. Acta Psychiatr Scand 1983; 67:361-70.

13. Drossman DA, Patrick DL, Whitehead WE, et al. Further validation of the IBS-QOL: a disease-specific quality-of-life questionnaire. Am J Gastroenterol 2000; 95:999-1007.

14. Rabin R, de Charro F. EQ-5D: a measure of health status from the EuroQol Group. Ann Med 2001; 33:337-43.

15. Bushnell DM, Martin ML, Ricci JF, Bracco A. Performance of the EQ-5D in patients with irritable bowel syndrome. Value Health 2006; 9:90-7.

16. Akehurst RL, Brazier JE, Mathers N, et al. Health-related quality of life and cost impact of irritable bowel syndrome in a UK primary care setting. Pharmacoeconomics 2002; 20:455-62.

17. Drossman D, Morris CB, Hu Y, et al. Characterization of health related quality of life (HRQOL) for patients with functional bowel disorder (FBD) and its response to treatment. Am J Gastroenterol 2007; 102:1442-53.

18. Shin S. ROC analysis for the evaluation of continuous biomarkers: Existing tools and new features in SAS® 9.2. Available at: http://pharmasug.org/ download/papers/SP09.pdf. Accessed November 21, 2013.

19. Mearin F, Badia X, Balboa A, et al; RITMO Group. Predictive factors of irritable bowel syndrome improvement: 1-year prospective evaluation in 400 patients. Aliment Pharmacol Ther 2006; 23:815-26.

20. Dobson A. An Introduction to Generalized Linear Models. London: Chapman \& Hall, 1990.

21. Kanazawa M, Endo Y, Whitehead WE, et al. Patients and nonconsulters with irritable bowel syndrome reporting a parental history of bowel problems have more impaired psychological distress. Dig Dis Sci 2004; 49:1046-53.

22. Creed F, Ratcliffe J, Fernandes L, et al; North of England IBS Research Group. Outcome in severe irritable bowel syndrome with and without accompanying depressive, panic and neurasthenic disorders. Br J Psychiatry 2005; 186:507-15.

23. Ringström G, Störsrud S, Posserud I, et al. Structured patient education is superior to written information in the management of patients with irritable bowel syndrome: a randomized controlled study. Eur J Gastroenterol Hepatol 2010; 22:420-8.

24. Heitkemper MM, Jarrett ME, Levy RL, et al. Self-management for women with irritable bowel syndrome. Clin Gastroenterol Hepatol 2004; 2:585-96.

25. Gwee KA, Wee S, Wong ML, Png DJ. The prevalence, symptom characteristics, and impact of irritable bowel syndrome in an asian urban community. Am J Gastroenterol 2004; 99:924-31.

26. Chang L, Toner BB, Fukudo S, et al. Gender, age, society, culture, and the patient's perspective in the functional gastrointestinal disorders. Gastroenterology 2006; 130:1435-46.

27. Department of Statistics, Singapore. Census of population 2013. Available from: http://www.singstat.gov.sg/publications/population.html\#census_of_ population. Accessed September 27, 2013.

28. Drossman DA, Li Z, Andruzzi E, et al. U.S. householder survey of functional gastrointestinal disorders. Prevalence, sociodemography, and health impact. Dig Dis Sci 1993; 38:1569-80.

29. Miwa H, Ghoshal UC, Fock KM, et al. Asian consensus report on functional dyspepsia. J Gastroenterol Hepatol 2012; 27:626-41. 


\title{
APPENDIX
}

\section{IRRITABLE BOWEL SYNDROME INFOPACK}

\author{
INFORMATION SECTION \\ - What is stress? \\ - How can stress affect Irritable Bowel Syndrome (IBS)?
}

\section{WHAT IS STRESS}

Stress affects one's thoughts, actions and body. Stress affects all sorts of people, anyone and everyone-young and old, male and female, rich or poor.

However, most people would rather have a 'real' problem like a broken leg than acknowledge their stress. It is important to know that you are not weak, mad or stupid because you have stress.

Just like how one can probably hide his/her stress from others, often people won't accept or are not happy to admit stress is a big problem in their lives. We all know that not everyone copes well with stress.

Often, the worst critic is you yourself. There is no magic cure for stress. Rather, this infopack teaches you to control stress, not get rid of it. As we said earlier, stress affects everyone and is part and parcel of life. So let's aim to manage it.

\section{HOW CAN STRESS AFFECT IBS?}

Stress can be broken up into three parts: T A B. T stands for Thoughts, A stands for Actions and B stands for Body.

When a person is under stress, he/she usually worries about things that might happen. For instance, "What if my stomach pain gets worse? What if it leads to something more serious?"

When a person is under stress, he/she usually engages in some actions such as avoiding (making decisions, taking responsibility, going out socially) and behaviours (checking too much, always rushed and trying to do too many things at one time).

When a person is under stress, he/she usually goes to their GP first complaining of physical symptoms. Our bodies respond to stress with a vast range of symptoms. Some people may be more prone than others to certain ones. Common bodily symptoms include rapid heart rate, sleeping problems, diarrhoea, constipation, and nausea.

Research has shown that IBS is related to heightened gut motility (muscle contraction), sensation, or both. The gut shares many nerve pathways and chemical transmitters with the brain.

While we now know IBS is not caused by stress, we know that stress may further aggravate bowel symptoms. Research has also indicated that if persons with IBS also suffer from depression and/or anxiety, their bowel symptoms may get worse during episodes of depression and anxiety. There is an interaction of various factors (such as biological make-up, childhood experiences and coping skills) causing one to be more likely than another to become stressed in the face of different environmental stressors. It is likely that IBS is due to an interaction between biological tendencies and environmental stressors. If an individual has a biological vulnerability to gastrointestinal disorders (e.g. hypersensitive gut), environmental factors such as food sensitivities, stressful life events that result in acute or chronic states of arousal can 'tip them over the edge', resulting in chronic bowel problems.

\section{TREATMENT SECTION}

- Quick control

- $\quad$ Additional resources

\section{QUICK CONTROL}

Managing stress is usually a long, slow business. Here are some quick ways to control stress: Distraction, Exercise, Talking and Breathing.

\section{Distraction}

Try to clear your mind as much as possible. Think of a word or phrase e.g. 'I am in control', 'Relax'. Close your eyes and slowly repeat the word/ phrase in your mind over and over again. Do this for ten minutes each day or whenever you are under stress. If unwanted thoughts come into your mind, just notice them, then redirect your attention back to the exercise.

\section{Exercise}

Studies have found that exercise helps to improve mood. It also helps you get out of the house and lets you meet new people.

\section{Talking}

Talking to a trusted friend or loved one often can allow you to release the stress.

\section{Breathing}

Place one hand on your chest and the other on your stomach (just above your belly button). As you breathe in through your nose, the hand on your stomach should be pushed out while the hand on your chest should not move. As you breathe out through your nose, your stomach should pull in. Your chest should not move.

We can also learn to put our stressful thoughts on trial. The first step is to identify your stressful thoughts. The second step is to look for evidence for the thoughts, then search for evidence against the thoughts. The final step is to sum up all the evidence and come to your own conclusions. IBS is not a life-threatening, chronic illness. It may recur over one's lifetime but it does not cause permanent harm to the intestines. For many people, having a proper diet and managing stress will help to alleviate the symptoms. 